\title{
A Review on Nanoparticles, Synthesis, Characterization, Current Applications and Future Perspectives
}

\author{
Sanwal Piracha ${ }^{1}$, Sohail Manzoor $^{2 *}$, Hareem Fatima ${ }^{2}$, Hira Aslam ${ }^{2}$, Muzammal Nazir ${ }^{1}$, Areeba Anjum ${ }^{1}$, Gulzaib \\ Basharat $^{1}$, Rikza Saif ${ }^{2}$ \\ ${ }^{1}$ Department of Chemistry, University of Agriculture Faisalabad, Pakistan \\ ${ }^{2}$ Department of Physics, University of Agriculture Faisalabad, Pakistan
}

*Corresponding author: Sohail Manzoor

\section{Abstract}

Nanotechnology playing significant role in different fields of sciences as the multidisciplinary particularly physics, chemistry, biology as well as in biomedical sciences. There are different types of nanoparticles depends upon on the particular metals and their function at cellular level. The most important nanoparticles are the carbon based, zinc and silver. Silver nanoparticles can be synthesized from silver nitrate as the main starting agent with sufficient quantity. One of the most important of synthesis of nanoparticles through green synthesis approach that are environment friendly. Through polysaccharides method, silver nanoparticles can be synthesized through the biological agent such as polysaccharides that particularly replaying impregnate role in characterization of nanoparticles in the presence of water. C. vulgaris as the active biological organism untitled for the production of silver nanoparticles at the normal room temperature. soluble polyoxometalates are capable of synthesizing noble nanoparticles through stepwise, multi-electron redox reactions inertly. TEM is a very powerful technique for the characterization of NP size, composition and crystalline structure. X-ray diffraction (XRD) is one of the most extensively used techniques for the characterization of NPs. Clay nanoparticles are playing important in textile industry to assist in making the long chain of fibers.

Keywords: Nanoparticles, synthesis, characterization, Nanotubes, nanotechnology.

Copyright () 2021 The Author(s): This is an open-access article distributed under the terms of the Creative Commons Attribution 4.0 International License (CC BY-NC 4.0) which permits unrestricted use, distribution, and reproduction in any medium for non-commercial use provided the original author and source are credited.

\section{INTRODUCTION}

Nanotechnology playing significant role in different fields of sciences as the multidisciplinary particularly physics, chemistry, biology as well as in biomedical sciences. It involves the synthesis of nanoparticles by different methods and technologies use in these fields to explore the discovery of novel cancer biomarkers as well as drug delivery to target the specific cells $[1,2]$. The ultimately goals of nanotechnology actually lies the discovery of new nanoparticles. Different sciences also synthesized different nanoparticles in response to particular metals included in them for their synthesis and characterization. The most important metals that are used for the synthesis of nanoparticles are the carbon, silvered based and zinc. Different methods are also used for their large scale production at the industrial level [35].

There are different types of nanoparticles depends upon on the particular metals and their function at cellular level. The most important nanoparticles are the carbon based, zinc and silver.

Nanoparticles in the form of carbon are employed in industries through chemical and physical methods. Then adopted into different shapes into spherical as well as cylindrical depend upon the temperature and catalysts used in their synthesis [6].

Metals are the most important part for synthesis of nanoparticles. Each metal playing significant role in catalysis and hence major significance in industries and medical equipment's for manufacturing of specific parts. They are most important nanoparticles in the form of zinc oxide. They are particularly synthesis in chemical and physical laboratories such as varnish and atomic absorption purpose. Nanoparticles in the form of aluminum are used for ceramics purposes to strengthen the rigidly of materials $[7,8]$. 


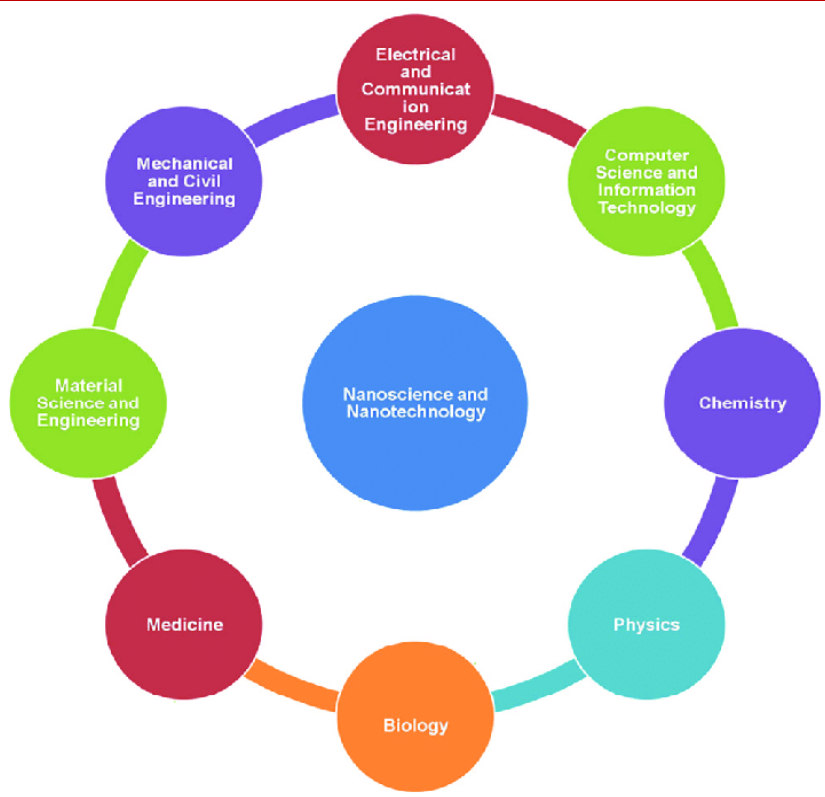

Fig-1: Shows the interrelationship of nanotechnology with branches of sciences

\section{Synthesis of nanoparticles}

Different types of biopolymers can be disintegrated into smaller nano blocks. These can be also further breakdown into small nanoparticles like the needles. This can be happens either through the physical methods or chemical methods that using the acid for their disintegration. The main advantage of small nanoparticles is their easy delivery to the cells for targeting them [9].

\section{Synthesis of Silver Nanoparticles}

Silver nanoparticles can be synthesized from silver nitrate as the main starting agent with sufficient quantity. Excess amount of silver nitrate leads to abnormal synthesis of silver nanoparticles that impure the final product. The other reagents and chemicals included the ascorbic acids 99\% and boric anhydride $250-500 \mathrm{mg} / \mathrm{dm} 3$. It can be synthesized as following the addition of boric anhydride in the form of drops to the silver nitrate using the ascorbic acid that ultimately leads the final product of silver nitrate nano particles $[10,11]$.

\section{Green synthesis of nanoparticles}

There are different methods for the synthesis of nanoparticles through chemicals and toxic substances but each of above method requires the large quality of chemicals that until yet leads to poor yield. These are not suitable for the controlled synthesis of nanoparticles rather they are produces toxin substances that pollutant the nanoparticles. Some conventional methods are not suitable for the production of nanoparticles due to high sots and low yield $[12,13]$.

One of the most important of synthesis of nanoparticles through green synthesis approach that are environment friendly. The nanoparticles that produced through this approach are high yield. To achieve the maximum yield of nanoparticles using greens approach, there are following methods used for their production at large scale as for the industrial level [14].

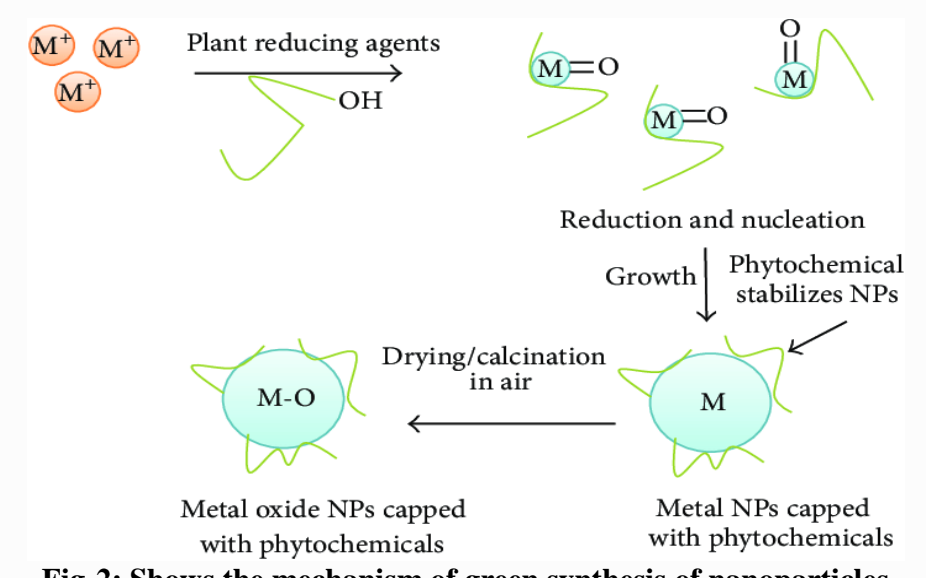

Fig-2: Shows the mechanism of green synthesis of nanoparticles 


\section{Polysaccharide method}

Through this method, silver nanoparticles can be synthesized through the biological agent such as polysaccharides that particularly replaying impregnate role in characterization of nanoparticles in the presence of water. It can be done in the presence of starch to protect the layer of the nano layer. This layer acts as the main biocatalyst to activate the reaction in chemical and physical processes. Damage of layer by starch leads to defective synthesis of silver nanoparticles that leads to poor yield [15].

\section{Polyoxometalates method}

Polyoxometalates are a vast family of molecular metal-oxide clusters with greater extent of structures. Meanwhile, their reduced forms possess greater capability of electron and proton transfer and/or storage abilities, and thus it can be employed to act as efficient donors or acceptors of several electrons without structural change. Hence, soluble polyoxometalates are capable of synthesizing noble nanoparticles through stepwise, multi-electron redox reactions inertly $[18,19]$.

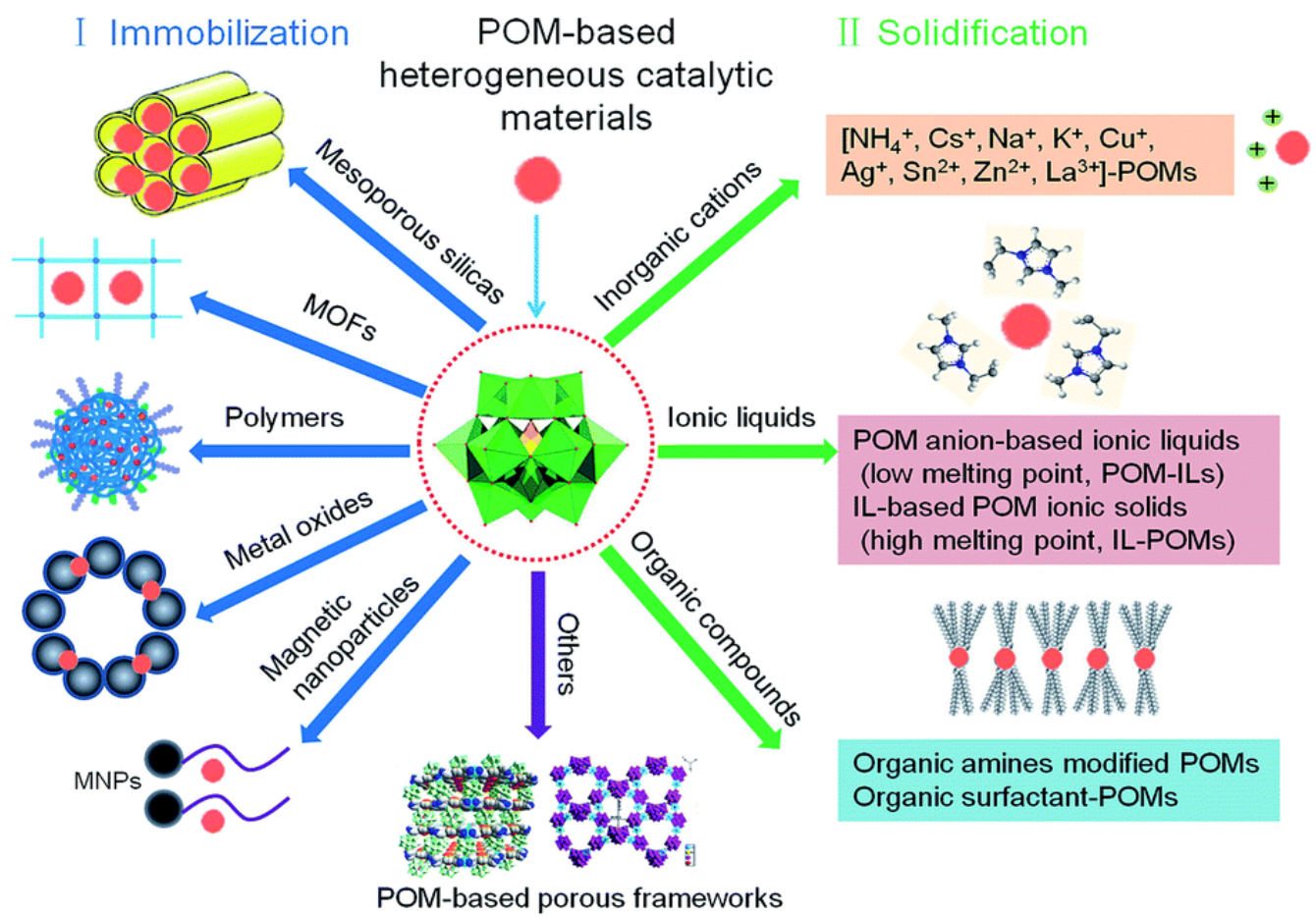

Fig-3: Shows the potential mechanism of polyoxometalate-based materials

\section{Biological method for nanoparticles}

C. vulgaris as the active biological organism untitled for the production of silver nanoparticles at the normal room temperature. Excess of temeperture ledas to defective synsthesid of silver nanoparticles. Different extracts from the microbes as well as the microbes can be employed for the synthesis of nanoparticles. Actually, the cells of living organisms utilized for the production of nanoparticles. Different biological agents such as proteins as well as metals that stabilized the reaction for synthesis of nanoparticles [16, 17].

\section{Nanoparticles characterization}

Once NPs are synthesized, it is important to fully characterize and understand their structure. Over the years, many methods have been developed for this purpose. In this section, the focus will be on the main techniques with relevance to this book, namely TEM, scanning TEM (STEM), electron energy loss spectroscopy (EELS) and x-ray photoelectron spectroscopy (XPS) [20].
TEM is a very powerful technique for the characterization of NP size, composition and crystalline structure. When an electron beam interacts with a sample, the electrons can be either transmitted, scattered, backscattered or diffracted [21,22]. TEM uses the transmitted electron signal to form an image of the sample. The transmitted electron beam is dependent on the sample thickness; for thin samples (a few nanometres), the transmitted electrons pass through without significant energy loss. STEM differs from TEM by focusing the electron beam into a narrow spot that is scanned over the sample in a raster [23].

X-ray diffraction (XRD) is one of the most extensively used techniques for the characterization of NPs. Typically, XRD provides information regarding the crystalline structure, nature of the phase, lattice parameters and crystalline grain size. The latter parameter is estimated by using the Scherrer equation using the broadening of the most intense peak of an XRD measurement for a specific sample. An advantage 
of the XRD techniques, commonly performed in samples of powder form, usually after drying their corresponding colloidal solutions, is that it results in statistically representative, volume-averaged values [24, 25].

X-ray absorption spectroscopy (XAS) includes both extended X-ray absorption fine structure (EXAFS) and X-ray absorption near edge structure (XANES, also known as NEXAFS). XAS measures the X-ray absorption coefficient of a material as a function of energy. Each element has a set of characteristic absorption edges corresponding to the different binding energies of its electrons, giving XAS element selectivity. As a highly sensitive technique, EXAFS is a convenient way to identify the chemical state of species which may occur even in very low concentrations [26, 27].

\section{Applications and effects physicochemical effects}

Clay nanoparticles are playing important in textile industry to assist in making the long chain of fibers. It also makes the dyeing process ideal before packaging into market. Polymers are the chains of smaller molecules that unite together in order to form the larger molecules. Nanoparticle acts at the central point of polymerization thus assassinating in polymerization. Nanoparticles also have applications in photolysis to carry the reactions of reduction as well as oxidation. Each nanoparticle has its own size that makes them functional to work at specific wavelength [28].

Silver nanoparticles can cause inflammatory bowel disease because of their reaction to immune system. If severe reaction occurs that leads to allergy and other inflammatory diseases. Many of nanoparticles that synthesized artificially can cause certain mutations in organism that untimely leads to certain defects in genome. One of the example of that health effects of nanoparticles on the human body is the silver nanoparticles [29, 30].

\section{CONCLUSION}

Metals are the most important part for synthesis of nanoparticles. Each metal playing significant role in catalysis and hence major significance in industries and medical equipment's for manufacturing of specific parts. Nanoparticles are useful for the drug delivery in one side while on the other hand, it may cause severe health issue if severe allergic reactions with high concentrations use for targeting the specific cells. We need to design such kind of nanoparticles that free from toxic effects on the cells and other delivery systems.

\section{REFERENCES}

1. Mohanraj, V. J., \& Chen, Y. (2006). Nanoparticles-a review. Tropical journal of pharmaceutical research, 5(1), 561-573.

2. Biswas, P., \& Wu, C. Y. (2005). Nanoparticles and the environment. Journal of the air \& waste management association, 55(6), 708-746.

3. Beydoun, D., Amal, R., Low, G., \& McEvoy, S. (1999). Role of nanoparticles in photocatalysis. Journal of Nanoparticle Research, 1(4), 439-458.

4. Schmid, G. (Ed.). (2011). Nanoparticles: from theory to application. John Wiley \& Sons.

5. Shahid, A., Ali, S., Zahra, T., Raza, M., Shahid, A., Saeed, M. U., \& Javaid, F. Influence of Microbes in Progression of Cancer and DNA Damaging Effects.

6. Iftikhar, A., Shahid, A., Shah, S. S., Ali, S., Raza, M., Ali, E., \& Umbreen, S. Antimicrobial Activities of Selected Medicinal Plant with Potential Role of Chemical Compounds.

7. Qamar, M., Mustafa, G. A., Tariq, S., Rafeeq, H., Rafiq, M., Naqvi, W. Z., ... \& Kanwal, T. Novel Methods for Detection of Biological Samples, Current Direction and Future Perspectives.

8. Naeem, M., Hayat, M., Qamar, S. A., Mehmood, T., Munir, A., Ahmad, G., ... \& Hussain, A. (2019). Risk factors, genetic mutations and prevention of breast cancer. Int. J. Biosci, 14(4), 492-496.

9. Shafiq, S., Adeel, M., Raza, H., Iqbal, R., Ahmad, Z., Naeem, M., ... \& Azmi, U. R. (2019). Effects of Foliar Application of Selenium in Maize (Zea Mays L.) under Cadmium Toxicity. In Biological Forum-An International Journal (Vol. 11, No. 2, pp. 27-37).

10. Ahmad, I., Khan, S., Naeem, M., Hayat, M., Azmi, U. R., Ahmed, S., ... \& Irfan, M. (2019). Molecular Identification of Ten Palm Species using DNA Fingerprinting. Int. J. Pure App. Biosci, 7(1), 46-51.

11. Usman, G., Muhammad, N., Hamza, R., Usman, I., Ayesha, A., Saqib, U., ... \& Fatima, Q. (2019). A Novel Approach towards Nutraceuticals and Biomedical Applications. Scholars International Journal of Biochemistry, 2(10), 245-252.

12. Tahir, M. F., Ali, S., Noman, M., \& Goher, M. A Novel Approach towards the Potential Effects of Chlorpyrifos on Testicular Biochemistry and Physiology of Male Sprague Dawely Rats.

13. Yang, W., Feng, H., Zhang, X., Zhang, J., Doonan, J. H., Batchelor, W. D., ... \& Yan, J. (2020). Crop phenomics and high-throughput phenotyping: past decades, current challenges, and future perspectives. Molecular Plant, 13(2), 187 214.

14. Naeem, M., Ali, J., Hassan, M. Z., Arshad, B., Rao, M. H. I., Sarmad, M. S. K., .. \&amp; Hassan, M. U. (2019). Novel Approach towards 
Sanwal Piracha et al., Sch Bull, Apr, 2021; 7(4): 118-122

DNA Barcoding as a Tool in Molecular Biologyand Biological Activities of Cyclotides with Particular Emphasizes at Molecular Level. In Biological Forum-An International Journal, 11(2), 83-96.

15. Naeem, A., Saddique, S., \& Chand, S. A. (2019). Advancement and Future Directions towards Herbal Treatment for Various Diseases.

16. De, M., Ghosh, P. S., \& Rotello, V. M. (2008). Applications of nanoparticles in biology. Advanced Materials, 20(22), 4225-4241.

17. Hosseinzadeh, K., Asadi, A., Mogharrebi, A. R., Azari, M. E., \& Ganji, D. D. (2021). Investigation of mixture fluid suspended by hybrid nanoparticles over vertical cylinder by considering shape factor effect. Journal of Thermal Analysis and Calorimetry, 143(2), 1081-1095.

18. Naeem, M., Ashraf, A., Safdar, H. M. Z., Khan, M. Q., Rehman, S. U., Iqbal, R., \& Ahmad, G. (2020). Biochemical changes in patients with chronic kidney failure in relation to complete blood count and anemia. IJB, 16(1), 267-271

19. Witwer, K. W., \& Wolfram, J. (2021). Extracellular vesicles versus synthetic nanoparticles for drug delivery. Nature Reviews Materials, 1-4.

20. Tang, S., Zhao, M., Yuan, D., Li, X., Zhang, X., Wang, Z., ... \& Wang, K. (2021). MnFe2O4 nanoparticles promoted electrochemical oxidation coupling with persulfate activation for tetracycline degradation. Separation and Purification Technology, 255, 117690.

21. Naeem, M., Hussain, A., Azmi, U. R., Maqsood, S., Imtiaz, U., Ali, H., ... \& Ghani, U. (2019). Comparative Anatomical Studies of Epidermis with Different Stomatal Patterns in Some Selected Plants Using Compound Light Microscopy. International Journal of Scientific and Research Publications, 9(10), 375-380.

22. Ahsan, M., Aslam, M., Akhtar, M. A., Azmi, U. R., Naeem, M., Murtaza, G., ... \& Shafiq, S. (2019). Effect of inoculation of three rhizobial strains on maize hybrids. Journal of Biodiversity and Environmental Sciences, 14(6), 168-177.
23. Hazafa, A., Batool, A., Ahmad, S., Amjad, M., Chaudhry, S. N., Asad, J., ... \& Ghani, U. (2020). Humanin: A mitochondrial-derived peptide in the treatment of apoptosis-related diseases. Life Sciences, Volume 264, 2021, 118679.

24. Rafeeq, H., Ahmad, S., Tareen, M. B. K., Shahzad, K. A., Bashir, A., Jabeen, R., ... \& Shehzadi, I. Biochemistry of Fat Soluble Vitamins, Sources, Biochemical Functions and Toxicity. Haya: The Saudi Journal of Life Sciences

25. Ghani, U., Naeem, M. Bukhari, S.S.H., Yar, G., Tariq, I., Siddique, S., Nawaz, H.A., Pal, Z.A.A., Nasim, F. and Bukhari, S.A.H. (2019). Prevalence and Risk Factors associated with Hepatitis B and Hepatitis C and their Correlation with Inflammatory Markers among Southern Region of Punjab. Biological Forum - An International Journal, 11(2): 136-143.

26. Muhammad Naeem, Umair Rasool Azmi,Sarmad Ahmad Qamar, Ahmad Munir, Tariq Mahmood, Muhammad Adeel Faryad. 2019. Reliable vaccine production by using Risk Based Bioengineering Strategies. 6th International Conference on "Sustainable Agriculture in Changing Climate: Strategies and Management. 261.

27. Rafeeq, H., Arshad, M. A., Amjad, S. F., Ullah, M. H., Muhammad, H., Imran, R. K., ... \& Ajmal, H. Effect of Nickel on Different Physiological Parameters of Raphanus Sativus.

28. Tang, S., Zhao, M., Yuan, D., Li, X., Zhang, X., Wang, Z., .. \& Wang, K. (2021). MnFe2O4 nanoparticles promoted electrochemical oxidation coupling with persulfate activation for tetracycline degradation. Separation and Purification Technology, 255, 117690.

29. Gao, Z., Shao, S., Gao, W., Tang, D., Tang, D., Zou, S., ... \& Xia, X. (2021). MorphologyInvariant Metallic Nanoparticles with Tunable Plasmonic Properties. ACS nano.

30. Litvin, A. P., Zhang, X., Ushakova, E. V., \& Rogach, A. L. (2021). Carbon Nanoparticles as Versatile Auxiliary Components of PerovskiteBased Optoelectronic Devices. Advanced Functional Materials, 2010768. 